\title{
Desempenho da produção agropecuária dos municípios pertencentes ao Escritório de Desenvolvimento Rural de Andradina, SP, Brasil
}

\author{
Agricultural production performance of municipalities belonging to Rural \\ Development Office Andradina, SP, Brazil

\section{Performance de la production agriculture des municipalités appartenant au Bureau de Développement Rural Andradina, SP, Brésil}

\author{
Rendimiento de la producción agrícola de los municipios pertenecientes a la \\ Oficina de Desarrollo Rural Andradina, SP, Brasil
}

\author{
Jaqueline Bonfim de Carvalho ${ }^{1}$ \\ Alan Rodrigo Panosso ${ }^{1}$ \\ Omar Jorge Sabbag ${ }^{1}$ \\ Maria Aparecida Anselmo Tarsitano ${ }^{1}$
}

Recebido em 31/10/2016; revisado e aprovado em 19/04/2017; aceito em 24/04/2017

DOI: http://dx.doi.org/10.20435/inter.v18i2.1432

\begin{abstract}
Resumo: A atribuição dos Escritórios de Desenvolvimento Rural (EDR) é execução das atividades relativas aos planos regionais e municipais de desenvolvimento rural. O objetivo do trabalho foi analisar a eficiência agropecuária de treze municípios pertencentes ao EDR de Andradina, SP, através da estatística multivariada e análise envoltória de dados (DEA). As análises permitiram concluir que a maioria das unidades trabalha de maneira ineficiente, tendo Valparaíso e Bento de Abreu como municípios destaque.
\end{abstract}

Palavras-chave: agrupamento; Andradina; eficiência agropecuária; estatística multivariada; DEA.

Abstract: The assignment of the Rural Development Offices (EDR) is execution of activities related to regional plans and municipal rural development. The objective of work was to analyze the agricultural efficiency thirteen municipalities belonging to EDR of Andradina/SP, by multivariate statistics and data envelopment analysis (DEA). The analyzes allowed conclude that most units working inefficiently, with Valparaíso and Bento de Abreu highlight municipalities.

Key words: grouping; Andradina; agricultural efficiency; multivariate statistics; DEA.

Résumé: La affectation des Bureaux de Développement Rural (EDR) est l'exécution des activités liées aux plans régionaux municipal et le développement rural. L'objectif travail était d'analyser l'efficacité de l'agriculture treize municipalités appartenant à EDR Andradina/SP, par les statistiques multivariées et l'analyse d'enveloppement des données (DEA). Les analyses ont montré que la plupart des unités fonctionne inefficacement, avec Valparaíso et Bento de Abreu comme les municipalités en vedete.

Mots-clés: regroupement; Andradina; efficacite agricole; statistiques multivariée; DEA.

Resúmen: La asignación de las Oficinas de Desarrollo Rural (EDR) es la ejecución de las actividades relacionadas con los planes regionales y municipales de desarrollo rural. El objetivo del trabajo fue analizar la eficiencia agrícola del trece municipios pertenecientes a la EDR Andradina/SP, por estadística multivariante y análisis envolvente de datos (DEA). Los análisis mostraron que la mayoría de las unidades trabaja de manera ineficiente, con Valparaíso y Bento de Abreu como los municipios destacados.

Palabras clave: agrupamiento; Andradina; eficiencia agrícola; estadística multivariante; DEA.

\section{INTRODUÇÃO}

Ao buscar estabelecer políticas que considerem as diversidades regionais, o Estado de São Paulo adotou, para fins de planejamento, uma divisão territorial em regiões. De maneira a atender as necessidades estratégicas de gestão de cada região, o Estado foi dividido em 40

\footnotetext{
${ }^{1}$ Universidade Estadual Paulista Júlio de Mesquita Filho (UNESP), Ilha Solteira, São Paulo, Brasil.
} 
Escritórios de Desenvolvimento Rural (EDR). Essa divisão do Estado em áreas de EDR foi definida a partir do Decreto no 41.559, criado em 1 o de janeiro de 1997, em que cada EDR possui a estrutura de um corpo técnico, um núcleo de apoio administrativo e casas da agricultura, sendo sua subordinação estabelecida de acordo com sua localização geográfica e características da produção agropecuária. A atribuição dos EDR's é planejar, coordenar e apoiar a execução das atividades relativas aos planos regionais e municipais de desenvolvimento rural, a extensão rural e assistência técnica, aos levantamentos e diagnósticos necessários à elaboração de políticas, a planos de desenvolvimento rural e outras necessidades do Governo do Estado, no âmbito da Coordenadoria de Assistência Técnica Integral (CATI) (SÃO PAULO, 1997).

O Estado de São Paulo é expressivo na agropecuária, em se tratando da produção agrícola, Caser et al. (2012), avaliando o valor da produção agropecuária do Estado para as 40 regiões, constataram que a cana-de-açúcar se destacou como principal produto, sendo o mais importante dentre 27 das 40 regiões do Estado, ou seja, com 70\% de predominância. Logo, em razão de a agropecuária ser uma dos setores da economia que mais empregam e geram excedente exportável, é importante analisar alternativas que possam refinar, ainda mais, a alocação dos recursos disponíveis.

Ademais, a eficiência nos sistemas de produção pode ser obtida de diversas formas, dentre estas por meio do manejo racional dos fatores de produção, como o uso da terra, o controle da mão de obra e o capital empregado na unidade agropecuária. Para qualquer situação, a utilização de tecnologia é responsável por incrementos importantes nos índices de produção (EUCLIDES FILHO, 2000). Nesse ponto de vista, a relevância de avaliar o emprego desses fatores de produção na região de estudo, avaliando a eficiência agropecuária dos municípios e os níveis tecnológicos que vêm sendo adotados, propondo melhorias quando necessário.

Uma ferramenta que o gestor pode utilizar é a Data Envelopment Analysis (DEA), que foi desenvolvida por Charnes e colaboradores, em 1978, e avalia unidades, com combinações de entradas e saídas, localizando a fronteira eficiente dentro de um grupo analisado, e que se tem mostrado atrativo em diversos setores de aplicação. O emprego de modelos DEA pode apoiar as decisões dos municípios correspondentes ao EDR de análise, ao indicar os municípios ineficientes e os que podem servir de referência às atividades agropecuárias (GOMES et al., 2003).

Outra forma de avaliação é a análise de agrupamento, com a finalidade de conhecer de antemão o comportamento dos dados. Essa análise visa explorar as similaridades entre unidades, definindo-as em grupos, considerando de maneira simultânea todas as variáveis notadas em cada unidade. Sendo uma análise multivariada, é um procedimento que identifica grupos em dados multivariados (multidimensionais) que são métodos de agrupamentos que classificam seus elementos em grupos restritos, homogêneos internamente, permitindo determinar tipologias analíticas e estruturas agregadas significativas (HÄRDLE; SIMAR, 2007; SIMÕES, 2003).

Com a informação relativa ao número de grupos e às características das variáveis utilizadas pelas unidades, a análise da eficiência produtiva permite considerar as especificidades tecnológicas e limitações para mudanças no sistema produtivo de uma unidade agropecuária. A análise multivariada, junto com a análise de eficiência, já vem sendo utilizada; Sousa et al. (2012), estudando o desempenho técnico dos produtores de leite no Estado de Goiás, empregaram a estatística multivariada para formação de grupos de produtores e o modelo de análise DEA para a estimativa de escores de eficiência e identificação de pontos de referência.

No presente trabalho, estudou-se um "recorte temporal", buscando verificar a eficiência dos municípios pertencentes ao EDR de Andradina, localizada na região oeste do Estado. A EDR 
de Andradina vem sofrendo na dinâmica produtiva do setor agropecuário, incluindo a substituição das áreas de pastagens, que se tornaram promissoras para a expansão do setor canavieiro, a presença de assentamentos e reassentamentos em seus municípios, com o predomínio da agricultura familiar. Assim, no intuito de propor medidas quanto ao uso eficiente dos principais fatores de produção, o objetivo do trabalho foi analisar a eficiência agropecuária de treze municípios pertencentes ao EDR de Andradina, por meio da análise multivariada e envoltória de dados.

\section{MATERIAL E MÉTODOS}

Os dados utilizados nesta pesquisa foram provenientes do banco de dados do site do Instituto Brasileiro de Geografia e Estatística (IBGE) - cidades, em relação ao censo agropecuário 2006, e do site da CATI - portal Levantamento Censitário das Unidades de Produção Agropecuária do Estado de São Paulo (LUPA), como referência o ano agrícola de 2007/2008 (SÃO PAULO, 2008).

A amostragem foi delimitada ao EDR de Andradina, região Noroeste do Estado de São Paulo, sendo composta por treze municípios, dentre eles: Andradina, Bento de Abreu, Castilho, Guaraçaí, Ilha Solteira, Itapura, Lavínia, Mirandópolis, Murutinga do Sul, Nova Independência, Pereira Barreto, Suzanápolis e Valparaíso.

Os municípios foram considerados as unidades produtivas, em que cada um representa uma Decision Making Units (DMU ou unidades tomadoras de decisão) para a medida de eficiência. Cada DMU realiza tarefas similares e se diferenciam nas quantidades de inputs que consomem e de outputs que produzem. De acordo com Macedo e Macedo (2003) que complementam a definição do termo DMU, essa será definida como uma organização, departamento, ou unidade administrativa, cuja eficiência está sendo analisada. Em uma avaliação DEA, o conjunto de DMU adotados deve ter em comum a utilização das mesmas entradas e saídas, ser homogêneas e ter autonomia na tomada de decisões. No que se referem às variáveis, cada uma destas deve trabalhar na mesma unidade de medida em todas as DMU, mas pode estar em unidades diferentes das outras.

Ao todo foram utilizados quatro variáveis para analisar a eficiência agropecuária da microrregião de Andradina. Dessas, três variáveis correspondentes aos insumos, ou seja, os inputs ( $\mathrm{X}$ = 3), sendo elas: terra, trabalho e capital. Para a variável produto, constituindo o output $(\mathrm{Y}=1)$ da análise, foi escolhido o valor produção agropecuária (mil reais). Todas essas quatro variáveis foram tomadas para cada município (DMU) analisado.

Para compor a variável terra, foi contabilizada a utilização das terras com lavouras permanentes, temporárias e pastagens, em hectare. De acordo com o IBGE (2006), as lavouras permanentes compreenderam a área plantada de culturas de longa duração, ou seja, culturas que, após a colheita, não necessitam de um novo plantio, produzindo por vários anos consecutivos. Já as lavouras temporárias abrangeram as áreas plantadas de culturas de curta duração (via de regra, menor que um ano) que necessitam usualmente de um novo plantio após a colheita. Para as pastagens, foram consideradas as áreas naturais (áreas destinadas ao pastoreio de gado, sem terem sido formadas mediante ao plantio), plantadas degradadas (por manejo inadequado ou por falta de conservação) e plantadas em boas condições (áreas destinadas ao pastoreio e formadas mediante plantio).

Em relação à variável trabalho, foi estabelecido o número de pessoal ocupado em estabelecimentos agropecuários, do sexo masculino. Essa categoria de gênero representou um maior número de pessoas quando comparado ao pessoal ocupado do sexo feminino. De acordo com o 
Dieese (2014), essa menor presença feminina na ocupação dos estabelecimentos rurais decorre tanto na predominância da família tradicional com chefia masculina, no caso da agricultura familiar, ou em relação à exigência de maior força física (geralmente associada aos homens), em diferentes atividades, cuja remuneração do trabalho é feita por produção.

Para a variável capital, foram consideradas as unidades de máquinas e implementos agrícolas utilizados em diversas atividades do setor agropecuário, e que fossem comuns para todos os municípios. Essa foi a única variável retirada do banco de dados do portal Lupa, as demais foram provenientes do banco de dados do IBGE cidades. As unidades de máquinas e implementos contabilizados foram: arado comum (bacia, aiveca), arado subsolador, desintegrador (picador/ triturador), distribuidor de calcário, grade aradora (tipo romi), grade niveladora, ordenhadeira mecânica, pulverizador tratorizado, semeadeira/adubadeira para plantio convencional, semeadeira/plantadeira para plantio direto e trator de pneus.

Inicialmente foram realizadas análises exploratórias multivariadas como ferramenta para elucidação da avaliação de eficiência investigada nesse trabalho, com o propósito de descrever e simplificar os dados a priori, identificando as relações entre as unidades (municípios) pertencentes ao EDR de Andradina. Para a análise multivariada, considerou-se cada DMU um acesso, sendo cada qual representada pelo valor das quatro variáveis (terra, trabalho, capital, valor da produção agropecuária) descritas posteriormente na avaliação de eficiência, formando assim uma matriz de treze linhas (acessos) por quatro colunas (variáveis inputs e output). Os dois métodos estatísticos multivariados aplicados foram: análise de agrupamentos hierárquicos e análise dos componentes principais. Para proceder às análises multivariadas, foi necessária a padronização das variáveis, em que cada uma ficou com a média zero e a variância 1.

A análise de agrupamentos hierárquicos (SNEATH; SOKAL, 1973) foi realizada calculando-se a distância euclidiana entre os acessos, para o conjunto das quatro variáveis, e para obtenção dos agrupamentos de acesso similares, sendo utilizado o algoritmo de Ward de variância mínima. Esse método tem como cálculo da distância a soma de quadrados entre os dois grupos feitos sobre todas as variáveis. Em cada estágio do procedimento de agrupamento, a soma interna de quadrados foi minimizada sobre todas as partições que podem ser obtidas pela combinação de dois grupos do estágio anterior. O objetivo principal dessa análise foi repartir os indivíduos em grupos homogêneos, de modo que cada grupo seja bem diferenciado. A análise de componentes principais (ACP) foi empregada para sintetizar a maior quantidade da informação original contida em $p$ variáveis $(p=4)$ em duas variáveis latentes ortogonais, denominadas componentes principais, que são combinações lineares das variáveis originais criadas com os dois maiores autovalores da matriz de covariância dos dados (HAIR, 2005). Dessa maneira, o conjunto inicial de quatro variáveis passou a ser caracterizado por duas novas variáveis latentes, o que possibilitou sua localização em figuras bidimensionais, formando um gráfico biplot. O ajuste dessa análise foi verificado pela quantidade da informação total das variáveis originais guardada pelos componentes principais que mostram autovalores superiores à unidade (KAISER, 1958).

Na segunda etapa do trabalho, foi realizada avaliação de eficiência por meio da Análise Envoltória de Dados (Data Envelopment Analysis [DEA]) para classificar as DMU's em eficientes e ineficientes. Com base nos conceitos de fronteiras eficientes da metodologia DEA para cada DMU, pode-se formular um modelo baseado em uma reta (modelo CCR) ou uma curva (modelo BCC). No primeiro caso, permite-se uma avaliação objetiva da eficiência global, além de possibilitar a identificação das fontes e viabilizar estimativas de montantes das ineficiências identificadas 
(KASSAI, 2002). Já no segundo caso, o BCC propõe uma restrição ao CCR e possibilita a determinação da eficiência de escala, identificando assim a presença de ganhos de escala crescentes, decrescentes e constantes (KASSAI, 2002; SOUZA; WILHELM, 2009).

A formulação matemática do modelo BCC-output utilizado no presente estudo pode ser expressa da seguinte forma:

$$
\begin{gathered}
\max h_{\circ} \\
\text { sujeito à } \\
x_{i o} \geq \sum_{k=1}^{n} x_{i k} \lambda_{i k} \forall i, \\
h_{\circ} y_{j \circ} \leq \sum_{k=1}^{n} y_{j k} \lambda_{k}, \forall j, \\
\lambda_{k} \geq 0, \forall k .
\end{gathered}
$$

Em que: $h_{0}$ é a medida radial de eficiência técnica; $y_{j}$ é o valor do output i; $x_{j}$ é o valor do input j; I é a importância da DMU k como referência para DMU 0.

Ainda em relação ao DEA, essa também pode ser classificada de acordo com a orientação desejada, seja input ou output. No presente trabalho, a orientação foi pelo output (maximização de produtos disponíveis, sem alteração no nível de insumos), supondo-se que os municípios são capazes de utilizar várias combinações dos recursos produtivos, podendo obter resultados diferenciados.

Para que a análise realizada pela técnica DEA tenha resultado satisfatório, é necessário que o número de unidades de produção seja, pelo menos, duas vezes o número de insumos $(X)$ e produtos $(Y)$, ou seja, $\geq 2(X+Y)$, encaixando-se perfeitamente na definição de DMU's (ALI; SEIFORD, 1993). Baseando-se nesse critério, treze unidades (DMU's) foram consideradas suficientes para que a análise tenha resultado satisfatório. Para a análise DEA foi utilizado o software DEAP (Data Envelopment Analysis Program), versão 2.1 (COELLI, 1996). As análises estatísticas foram conduzidas no programa STATISTICA 7.0 (STATSOFT, 2004).

\section{RESULTADOS E DISCUSSÃO}

Inicialmente são apresentadas a relação de inputs e output avaliados na análise multivariada e DEA e a estatística descritiva dessas variáveis (Tabela 1). Os resultados mostraram que a média da utilização da terra foi 38.313 ha, com mediana de 31.687 ha e uma amplitude de variação de 63.811 ha por município entre os valores mínimo e máximo. Isso nos dá um indicativo para possíveis diferenças entre as unidades analisadas. 
Tabela 1 - Relação de inputs e outputs e estatística descritiva dessas variáveis, correspondentes aos municípios (DMU's) pertencentes ao EDR de Andradina

\begin{tabular}{lccccccc}
\hline Variáveis & Unidade & Média & Mediana & Desvio Padrão & Mínimo & Máximo \\
\hline Terra & Hectare & 38.313 & 31.687 & 19.094 & 13.300 & 77.111 \\
Trabalho & No pessoas s.m. & 1.204 & 1.134 & 851 & 219 & 3.164 \\
Capital & No maq. e imple. & 851 & 704 & 577 & 204 & 1.971 \\
\hline \multicolumn{7}{c}{ Output } \\
\hline $\begin{array}{l}\text { Produção (Valor } \\
\text { da produção) }\end{array}$ & \multirow{2}{*}{ Mil reais } & 27.896 & 19.306 & 40.922 & 2.200 & \multirow{2}{*}{152.947} \\
\hline
\end{tabular}

* Número de pessoas do sexo masculino;

** Número de máquinas e implementos.

Fonte: A partir de dados primários do IBGE (2006) e Portal LUPA (2007/2008).

Para a variável trabalho, o número médio de pessoal ocupado em estabelecimentos agropecuários do sexo masculino foi de 1.204 pessoas, e mediana próxima de 1.134 pessoas, com uma diferença de 2.945 pessoas por município entre os valores mínimo e máximo, também evidenciando um distanciamento entre as unidades analisadas. Essa diferença no número de pessoas que realizam a mão de obra pode ser justificada devido às modificações tecnológicas que a agropecuária sofreu nas últimas décadas após a "revolução verde". Segundo Vian e Júnior (2010), o surgimento desse novo setor mudou o rumo das técnicas de produção e oferta de produtos agrícolas no mundo, assim como a necessidade de envolvimento de mão de obra na produção agrícola, pois o aumento da produtividade do setor levou à substituição do homem nessa atividade, possibilitando o acesso a melhores práticas de produção na agricultura e pecuária.

De acordo com Alisson e Arantes (2016) a produção da agricultura paulista aumentou em mais de $90 \%$ nas últimas décadas. Os autores citam que houve uma redução no número de trabalhadores cerca de 30\% entre o censo agropecuário de 1970 e o de 2006. Isso ocorreu devido ao deslocamento da população de origem rural para os centros urbanos. Os autores ainda citam que a redução do pessoal ocupado não teve reflexo negativo sobre a produção e a produtividade. "Trata-se de uma tendência considerada 'normal' nas regiões desenvolvidas, nas quais menos pessoas produzem mais, devido ao maior aporte de tecnologia". Essa menor quantidade de trabalhadores se dá pelo aumento da mecanização nas lavouras paulistas.

O mesmo distanciamento pode ser observado em relação a variável capital, que se refere ao número de máquinas e implementos agrícolas utilizados nas unidades. Nota-se uma variação de 1.767 entre os valores mínimo e máximo, evidenciando que, nesse quesito, existem grandes diferenças do nível tecnológico entre as unidades analisadas.

Para a variável produção foi considerado o valor da produção comercializada, com média de $\mathrm{R} \$ 27.896$ mil reais, mediana de $\mathrm{R} \$ 19.306$ e amplitude de $\mathrm{R} \$ 150.747$ mil reais. Nessa variável, nota-se que a mediana não se aproxima da média como nas variáveis anteriores, ou seja, a média pode estar sendo influenciada por valores "muito grandes" ou "muito pequenos". Pode-se dizer que está enviesada para a direita (possui valores grandes como "outliers"), pois a média tende a ser maior que mediana. O valor máximo que está influenciando a média pertence à unidade de Valparaíso o qual obteve o maior valor da produção (R\$ 152.947 mil reais), tendo a cana-de-açúcar como seu principal produto comercializado. As demais unidades apresentaram valores 
abaixo, tendo a unidade de Itapura o menor valor de produção quando comparada às demais unidades, em que o principal produto comercializado por essa DMU foi o feijão.

Analisando os dados gerais de valores de produção de cada município pertencente ao EDR de Andradina, é possível observar que os maiores valores de produção são de culturas como cana-de-açúcar, milho e feijão, além de produtos de origem animal como o leite de vaca e ovos de galinhas. Segundo Alisson e Arantes (2016), a agricultura paulista é concentrada em apenas alguns produtos. A cana-de-açúcar contribuiu com 59,3\% seguida pela laranja com 12,6\% do valor da produção agrícola no triênio de 2010 a 2012. Por mais que laranja não possua grande relevância na região de estudo, tem seu destaque no cenário paulista. Porém, vale lembrar que os autores ressaltam que há de se considerar que essas estatísticas englobam as grandes lavouras. Contudo ressalta-se que os municípios paulistas possuem floricultura, horticultura e algumas frutíferas, sendo culturas com expressividade no Estado.

Após a estatística descritiva, uma estatística multivariada foi realizada com o caráter exploratório dos dados. No dendrograma (Figura 1) é possível reconhecer dois agrupamentos, considerando o corte a uma distância euclidiana igual a 7. O primeiro cluster, no canto esquerdo superior da imagem, constituiu os acessos Andradina, Mirandópolis, Castilho, Guaraçaí, Pereira Barreto e Valparaíso. Nota-se que Valparaíso não possui sub-agrupamento, indicando que exista alguma diferença entre os outros acessos; diferente, por exemplo, de Andradina e Mirandópolis, que pertencem ao mesmo grupo de Valparaíso, porém são agrupados anteriormente por um sub-agrupamento indicando similaridade entre eles.

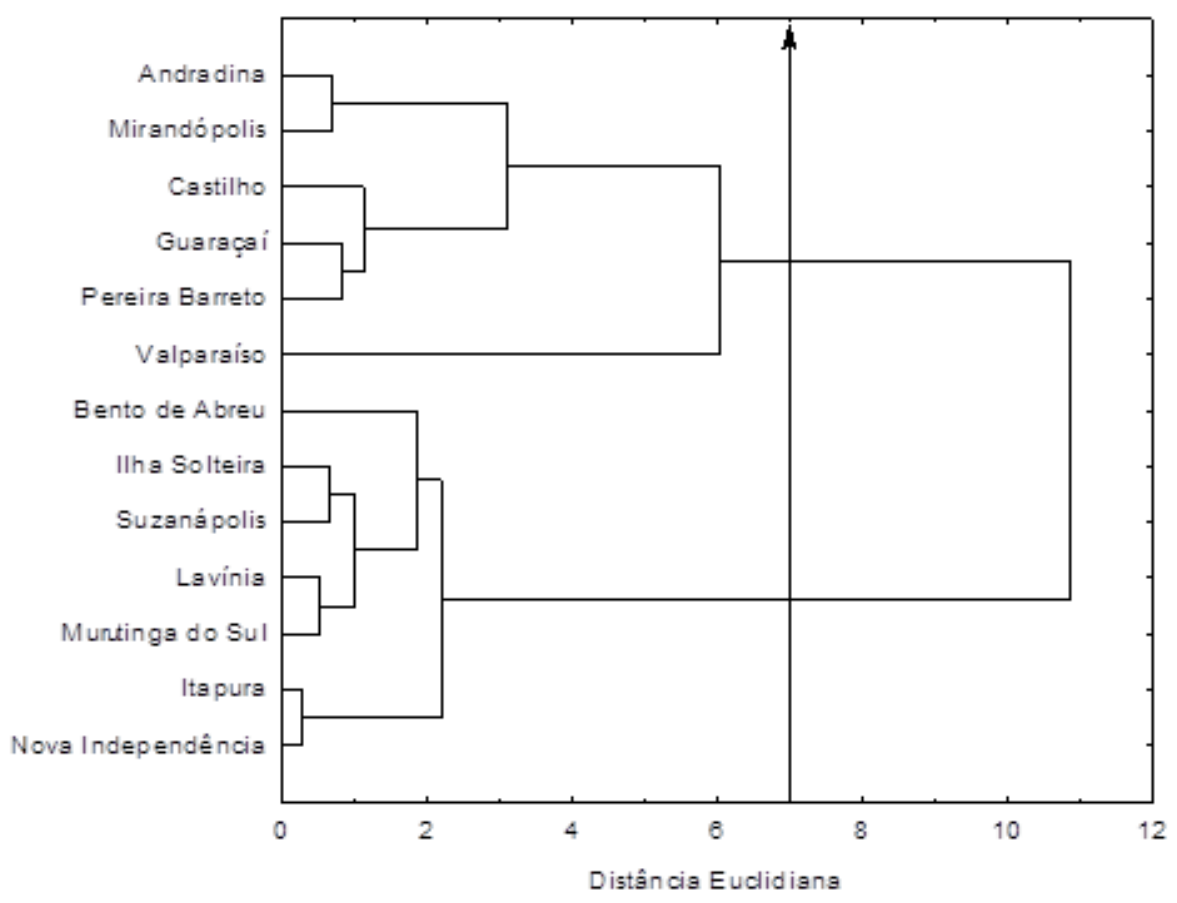

Figura 1 - Dendrograma resultante da análise hierárquica de agrupamentos. Fonte: A partir de dados primários do IBGE (2006) e Portal LUPA (2007/2008).

De acordo com Metz (2006), bons agrupamentos são compactos e apresentam alta similaridade, e uma maneira de verificar essa compactação é analisando a altura dos arcos que agrupam os clusters, pois quanto menor a sua altura, mais compactos eles serão. Por outro 
lado, a junção entre clusters distintos deve apresentar pouca similaridade, portanto o arco que os une deve ser maior em relação aos arcos que conectam seus subagrupamentos. O segundo agrupamento formado foi constituído pelos acessos Bento de Abreu, Ilha Solteira, Suzanápolis, Lavínia, Murutinga do Sul, Itapura e Nova Independência. Os arcos que os unem mostram que existe maior similaridade entre os acessos do segundo cluster quando comparados ao primeiro, devido à menor altura dos arcos que os conectam.

Os resultados apresentados pelo dendrograma são reforçados pela ACP, por meio do gráfico biplot entre os dois primeiros componentes principais (Figura 2). O componente que reteve mais informação (maior variabilidade das variáveis originais) é o componente principal 1 (CP1 $=71,08 \%$ ). Os dois primeiros componentes principais juntos (CP1 e CP2) captaram 96,44\% da variabilidade total do conjunto de dados originais. Dentro do CP1, as variáveis que apresentam o maior poder discriminatório, em ordem de importância, foram: terra (correlação $=0,98$ ), trabalho (correlação $=0,97$ ) e produção (correlação $=0,70$ ). Como as correlações entre CP1 são positivas, indicam que unidades mais à direita sofrem maior influência dessas características. $O$ sinal indica direção positiva ou negativa do relacionamento, e o valor sugere a força da relação entre as variáveis. Valores mais próximos de 1 (independente do sinal), maior é o grau de dependência linear entre as variáveis.

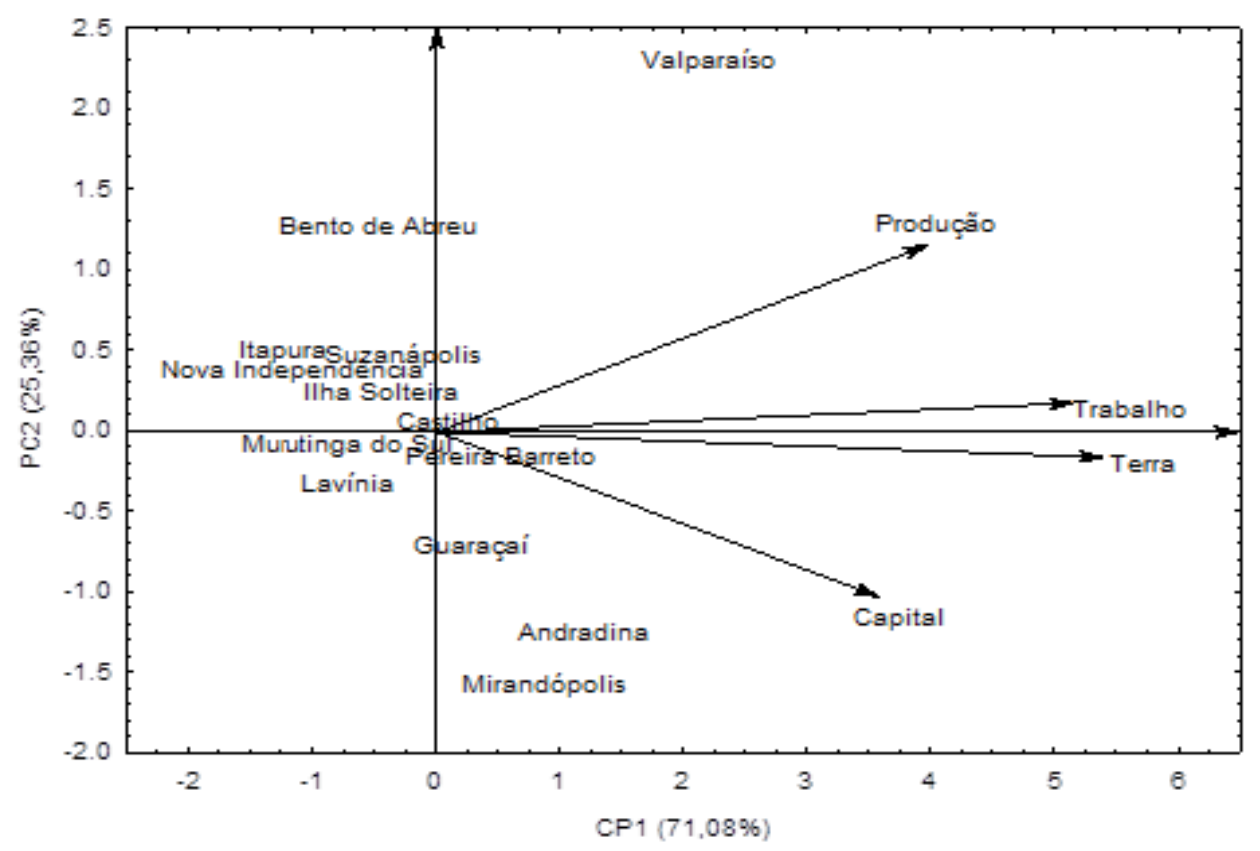

Figura 2 - Resultado da análise de componentes principais, gráfico de dispersão biplot entre o primeiro (CP1) e o segundo (CP2) componentes principais. Fonte: A partir de dados primários do IBGE (2006) e Portal LUPA (2007/2008).

Valparaíso se destacou dos demais acessos, sendo influenciada pela variável produção, trabalho e terra. Segundo dados do Portal LUPA (2007/2008), esse município possuía uma área de 43.627 hectares com a cana-de-açúcar, sendo esta sua principal cultura. Possivelmente esse foi um fator para que esse acesso se destacasse dos demais. Analisando o avanço da cana-de-açúcar no Estado de São Paulo em relação às outras culturas, no período de 2001 a 2006 Camargo et al. (2008) observaram que para a região oeste paulista, tradicional na pecuária de corte apresentou 
uma retração da área plantada com pastagem cultivada, em que a cana-de-açúcar incorporou cerca de $75,37 \%$ da área de pastagem na EDR de Andradina. Para a região oeste, somente no período da pesquisa, a área cedida por pastagem cultivada equivaleu a 619.380 ha, significando que, de toda área entregue por essa atividade no Estado de São Paulo, 62\% concentraram-se nesse espaço. Contudo muitos dos agricultores que mudaram para a atividade canavieira, não possuem máquinas e implementos adequados ao setor (KANEKO et al., 2009).

A CP2 apresentou a variável capital com maior poder discriminatório, com correlação negativa $=-0,74$ aos acessos na parte inferior do gráfico biplot. A unidade de Murutinga do Sul foi influenciada por essa componente, significando que possui um maior número de máquinas e implementos agrícolas. De acordo com o portal LUPA (2007/2008), a cultura que possui maior destaque no município é a braquiária com $16.723,3$ ha, porém, confrontando com dados do Censo Agropecuário (2006), foi observado que o valor da produção de leite de vaca, que é uma atividade derivada de produtores alicerçados em culturas forrageiras, no ano nos estabelecimentos agropecuários é de $\mathrm{R} \$ 1.391,00$. Esse valor de produção acaba não sendo expressivo, quando comparado ao acesso que obteve mais destaque (Valparaíso) em que o valor da produção de cana-de-açúcar acaba sendo superior ao de leite de vaca, motivo pelo qual esse acesso não se sobressaísse em relação aos demais.

Na Tabela 2 são apresentados os valores de eficiência e a natureza dos retornos das DMU's. Para a eficiência com retornos variáveis de escala (BCC), as DMU's que atingiram 100\% foram as unidades Bento de Abreu, Itapura, Nova Independência e Valparaíso. Entretanto, quando se analisa sua escala e natureza dos retornos, apenas Bento de Abreu e Valparaíso atingiram retornos constantes. Isso significa que há um equilíbrio entre inputs e output operando em suas capacidades ótimas.

Tabela 2 - Valores de eficiência e natureza dos retornos das DMU's pertencentes ao EDR de Andradina

\begin{tabular}{ccccc}
\hline DMU & CCR & BCC & Escala* & Natureza dos retornos \\
\hline Andradina & 0,19 & 0,20 & 0,93 & decrescente \\
Bento de Abreu & 1,00 & 1,00 & 1,00 & constante \\
Castilho & 0,18 & 0,23 & 0,77 & decrescente \\
Guaraçaí & 0,23 & 0,24 & 0,96 & decrescente \\
Itapura & 0,08 & 1,00 & 0,08 & crescente \\
Ilha Solteira & 0,06 & 0,06 & 0,99 & decrescente \\
Lavínia & 0,11 & 0,12 & 0,96 & crescente \\
Mirandópolis & 0,10 & 0,11 & 0,92 & decrescente \\
Murutinga do Sul & 0,10 & 0,14 & 0,70 & crescente \\
Nova Independência & 0,10 & 1,00 & 0,10 & crescente \\
Pereira Barreto & 0,27 & 0,29 & 0,93 & decrescente \\
Suzanápolis & 0,37 & 0,40 & 0,92 & crescente \\
Valparaíso & 1,00 & 1,00 & 1,00 & constante \\
\hline
\end{tabular}

*Eficiência de Escala: CCR/BCC

Fonte: A partir de dados primários do IBGE (2006) e Portal LUPA (2007/2008).

De acordo com Carvalho et al. (2016), quando unidades atingem níveis constantes em relação ao retorno, essas possuem os níveis ótimos ou metas (targets) de utilização das variáveis em 
questão, valores que as demais DMU's devem atingir, isto é, as unidades com retornos constantes servem como referência para a projeção dos demais municípios ineficientes. No caso da análise de eficiência, as unidades modelo seriam aquelas que atingiram retorno constante de escala. Ressalta-se que as DMU's que atingiram natureza constante dos retornos possui sua atividade agrícola voltada quase que exclusivamente a cultura da cana-de-açúcar.

Bento de Abreu e Valparaíso possuem usinas do grupo Raízen, que se destaca como uma das empresas de energia mais competitivas do mundo. Em Valparaíso se localiza a usina Univalem, a primeira destilaria do oeste do Estado em 1978. Já Bento de Abreu abriga a usina Benálcool, que inicialmente teve a produção de álcool hidratado a extraído da cana-de-açúcar. Teve a incorporação a partir de 1996 a produção de açúcar. Ambos os municípios se localizam as maiores áreas produtoras de cana-de-açúcar na região (NOVA CANA, 2016; PROENÇA, 2008). Caracterizando a produção de cana-de-açúcar e as inovações tecnológicas adotadas por usinas da EDR de Andradina, no período de 2003/04 a 2006/07 Proença (2008) encontrou uma produtividade média de $83 \mathrm{t} \mathrm{ha}^{-1}$, tendo Valparaíso com uma produtividade média de 80 t ha ${ }^{-1}$ e Bento de Abreu, por sua vez, que possui uma área menor que Andradina, conseguiu ter uma produtividade média de $85 \mathrm{t} \mathrm{ha}^{-1}$, enquanto Andradina possui uma produtividade de $80 \mathrm{t} \mathrm{ha}^{-1}$. Esses municípios apresentaram produtividades abaixo da média do EDR devido ao fato de não serem oriundas de novas áreas, que por sua vez conseguem apresentar maiores produtividades.

Porém os resultados da análise DEA indicam que a DMU Andradina não atingiu eficiência, tendo seu retorno de escala decrescente, junto às DMU's Castilho, Guaraçaí, Ilha Solteira, Mirandópolis, e Pereira Barreto. Essas DMU's operam em escala "supraótima", ou seja, quando ocorre desperdício no uso dos insumos (terra, trabalho, capital) para que se obtenha o mesmo retorno produtivo. Pode-se observar que a cultura de destaque para todas essas DMU's, que operaram em escala decrescente, foi a braquiária, indicando a presença de pastagem (LUPA, 2007/2008). Segundo Borges e Mateus (2012), a região de oeste do Estado de São Paulo possui mais de um milhão de hectares de pastagens, sendo que aproximadamente $70 \%$ dessa área se encontram em processo de degradação com uma área potencial a ser reformada. Os autores ainda reforçam que uma alternativa para a recuperação dessas áreas, seria a integração agricultura e pecuária. Um exemplo seria colocar 25\% dessa área de pastagem degradada, áreas que atualmente produzem muito pouco, e, em médio prazo, essas áreas poderiam estar produzindo 3,82 milhões de toneladas de grãos e 2,3 milhões toneladas em carcaça de carne bovina.

Os resultados encontrados neste trabalho com baixos escores de eficiência foram semelhantes ao proposto por Lopes (2014), que, avaliando a ecoeficiência na agropecuária nos municípios brasileiros da região Norte através da análise DEA, e utilizando como inputs pessoal ocupado, área total agrícola, insumos agropecuários e capital estimado pela depreciação, e, como output a variável, valor total da produção agropecuária do município, e a partir também de dados do Censo Agropecuário de 2006, concluiu que apenas 5,7\% dos municípios analisados foram eficientes. Cita ainda que, o fator terras degradadas foi o que mais prejudicou os municípios ineficientes, tendo que haver uma diminuição desse problema para que estes pudessem se destacar.

As DMU's que atingiram retorno crescente de escala foram: Itapura, Lavínia, Murutinga do Sul, Nova Independência e Suzanápolis. Essas unidades trabalharam na faixa "subótima", ou 
seja, abaixo da faixa ótima, necessitando de estímulos dos insumos para aumento da produção. Essas unidades possuem assentamentos em suas composições agropecuárias, em que se observam a realização de várias atividades (mandioca, milho, feijão, banana e leite de vaca), além da presença da cultura da cana-de-açúcar próxima às unidades, com um maior nível tecnológico aplicado às áreas.

De acordo com Silva (2012), estudando aspectos da Microrregião de Andradina, existe a presença de policultura e a exploração de culturas com custos de produção relativamente baixos e sem exigência de infraestrutura específica. Porém enfrentam fortes limitações relacionadas com a precariedade da infraestrutura disponível, limitações de crédito e com baixa efetividade e qualidade, predominante em grande parte dos serviços de assistência técnica e extensão rural. Uma saída para os produtores é a exploração de circuitos curtos de comercialização, comuns entre os pequenos agricultores da Microrregião. Darolt, Lamine e Brandemburg (2013) citam que circuitos curtos de comercialização proporcionam não somente ganhos em escala (quantidade), mas em qualidade. Além de criar novas relações sociais, promove o resgate da autonomia dos agricultores, que pode ser de grande importância para os produtores da região avaliada. Os autores também ressaltam o papel fundamental das políticas públicas no sentido de formar e informar os consumidores menos esclarecidos.

Logo, podemos afirmar, com base nas duas análises exploratórias dos dados, assim como a análise de eficiência, que a região de Andradina, mais precisamente os municípios pertencentes ao EDR de Andradina possuem níveis tecnológicos diferentes, devido às diferentes culturas presentes nas unidades, ou seja, aquelas que obtiveram destaque possuem, quase que exclusivamente, a cana-de-açúcar como a principal atividade agrícola, além da presença de pastagem; por outro lado, algumas áreas se encontram em processo de degradação.

\section{CONSIDERAÇÕES FINAIS}

A estatística multivariada, junto com a análise DEA, permitiu observar que apenas 30,77\% de treze municípios trabalharam de maneira eficiente no modelo BCC, e apenas dois municípios operam com retornos constante de escala, sendo Bento de Abreu e Valparaíso, que atingiu níveis ótimos da utilização das variáveis em questão, no período abordado. Para se buscar a eficiência nas outras unidades, é necessário que se tenha otimização das áreas, principalmente aquelas ocupadas por pastagens degradadas.

A cana-de-açúcar é a cultura de destaque nas unidades eficientes nessa análise, já que o trabalho se trata de um "recorte temporal" e retratou o início de parte de um processo de diferenciação de estrutura produtiva. Necessário realizar um estudo comparativo quando houver o novo Censo Agropecuário e novo LUPA para verificar o nível de incorporação das áreas de pastagem, principalmente pela cana-de-açúcar.

Outra sugestão para estudos futuros é que se incorpore um maior número de variáveis, para conseguir identificar diferentes níveis sociais e tecnológicos que existem nos municípios pertencentes ao EDR de Andradina.

\section{REFERÊNCIAS}

ALI, A. I., SEIFORD, L. M. The Mathematical Programming Approach to Efficiency Analysis. In: FRIED, H. O., LOVELL, C. A. K., SCHIMIDT, S. S. (Org.). The measurement of productive efficiency: techniques and application. New York: Oxford University Press, 1993. cap. 3, p. 120-159. 
ALISSON, E.; ARANTES, J. T. Produção da agricultura paulista aumenta em mais de $90 \%$ nas últimas duas décadas. São Paulo: Agência FAPESP, 2016. Disponível em: <http://agencia.fapesp.br/producao_da_ agricultura_paulista_aumenta_em_mais_de_90_nas_ultimas_duas_decadas/24008/>. Acesso em: 10 set. 2016.

BORGES, W. L. B.; MATEUS, G. P. Polos regionais noroeste paulista e extremo oeste: integração lavourapecuária. Pesquisa e Tecnologia, São Paulo, n. 1, edição especial, 2012.

CAMARGO, A. M. M. P.; CASER, D. V.; CAMARGO, F. P.; OlIVETTE, M. P. A.; SACHS, R. C. C.; TORQUATO, S. A. Dinâmica e tendência da expansão da cana-de-açúcar sobre as demais atividades agropecuárias, Estado de São Paulo, 2001-2006. Revista Informações Econômicas, São Paulo, v. 38, n. 3, p. 47-66, mar. 2008. Disponível em: < http://www.iea.sp.gov.br/out/LerTexto.php?codTexto=9237>. Acesso em: 15 set. 2008.

CARVALHO, J. B.; MODENESE, V. S.; SANTANA, V. Z.; SABBAG, O. J. Análise de eficiência do Programa Aquisição de Alimentos para o território de Andradina e Noroeste Paulista. Revista Espacios, Caracas, v. 37, n. 10, ano 16, p. 16, 2016.

CASER, D. V.; TSUNECHIRO, A.; COELHO, P. J.; BUENO, C. R. F.; PINATTI, E.; CASTANHO FILHO, E. P.; BINI, D. L. Valor da produção agropecuária por região, Estado de São Paulo, 2011. Análises e Indicadores do Agronegócio, São Paulo, v. 7, n. 6, jun. 2012.

CHARNES, A.; COOPER, W. W.; RHODES, E. Measuring the efficiency of decision making units. European Journal of Operational Research, v. 2, p. 429-444, 1978.

COELLI, T. J. A guide to DEAP version 2.1: a data envelopment analysis program. Armidale, Austrália: University of New England, 1996. 49 p. (CEPA Working Papers, 08/96).

DAROLT, M.R.; LAMINE, C.; BRANDEMBURG, A. A diversidade dos circuitos curtos de alimentos ecológicos: ensinamentos do caso brasileiro e francês. Agriculturas, v. 10, n. 2 p. 8-13, 2013.

DEPARTAMENTO INTERSINDICAL DE ESTATÍSTICA E ESTUDOS SOCIOECONÔMICOS (DIEESE). O mercado de trabalho assalariado rural brasileiro. Estudos e pesquisas, n. 74, p. 1-33, out. 2014.

EUCLIDES FILHO, K. Produção de bovinos de corte e o trinômio genótipo-ambiente-mercado. Campo Grande: Embrapa Gado de Corte, 2000. 61 p. (Documentos, 85).

GOMES, E. G.; SOARES DE MELLO, J. C. C. B.; BIONDI, L. N. Avaliação de Eficiência por Análise de Envoltória de Dados: conceitos, aplicações à agricultura e integração com sistemas de informação geográfica. Campinas: Embrapa Monitoramento por Satélite, 2003. 39 p. (Documentos, 28).

HAIR, J. F.; ANDERSON, R. E.; TATHAM, R. L.; BLACK, W. Análise multivariada de dados. Porto Alegre: Bookman, 2005.

HÄRDLE, W.; SIMAR, L. Applied multivariate statistical analysis. 2. ed. Berlin: Springer, 2007. 486 p.

INSTITUTO BRASILEIRO DE GEOGRAFIA E ESTATÍSTICA (IBGE). Cidades@. Censo Agropecuário 2006, 2006. Disponível em: <http://www.cidades.ibge.gov.br/xtras/home.php> Acesso em: jan. 2016.

KAISER, H. F. The varimax criterion for analytic rotation in factor analysis. Psychometrika, v. 23, p. 187200, 1958.

KANEKO, F. H.; TARSITANO, M. A. A.; RAPASSI, R. M. A.; CHIODEROLI, C. A.; NAKAYAMA, F. T. Análise econômica da produção de cana-de-açúcar considerando-se a terceirização das operações agrícolas: o caso de um produtor. Pesquisa Agropecuária Tropical, v. 39, n. 3, p. 266-270, 2009.

KASSAI, S. Utilização da Análise Envoltória de Dados (DEA) na análise de demonstrações contábeis. 350f. Tese (Doutorado em Contabilidade e Controladoria) - Faculdade de Economia, Administração e Contabilidade, Universidade de São Paulo, São Paulo, 2002.

LOPES, B. A. G. Ecoeficiência na agropecuária: uma aplicação da Análise Envoltória de Dados- DEA nos municípios brasileiros da região Norte. 2014. 183 f. Dissertação (Mestrado em Agronegócio)- Faculdade de Agronomia e Medicina Veterinária, Universidade de Brasília, Brasília, DF, 2014.

MACEDO, M. A. S; MACEDO, H. D. R. Avaliação de performance financeira através da análise envoltória 
de dados: um estudo de caso em unidades de negócio. In: Congresso Latino-Americano de Escolas de Administração (CLADEA), 38., 2003, Lima, Peru. Anais... Lima, 2003.

METZ, J. Interpretação de clusters gerados por algoritmos de clustering hierárquico. 2006. 126f. Dissertação (Mestrado em Ciências de Computação e Matemática Computacional) - Instituto de Ciências Matemáticas e de Computação (ICMC), Universidade de São Paulo, São Carlos, SP, 2006.

NOVA CANA. Unidade benalcool. [s.l.; s.n.], 2016. Disponível em: <https://www.novacana.com/usinasbrasil/sudeste/sao-paulo/unidade-benalcool/>. Acesso em: 1ㅇs. 2016.

PROENÇA, E. R. Caracterização da produção de cana-de-açúcar e de inovações tecnológicas adotadas por Usinas da Regional de Andradina (SP). 2008, 69f. Dissertação (Mestrado em Agronomia) - Faculdade de Engenharia de Ilha Solteira, Universidade Estadual Paulista, Ilha Solteira, SP, 2008.

SÃO PAULO, Cidade [de]. Decreto n. 41.559, de 21 de janeiro de 1997. Dispõe sobre a estrutura administrativa da Coordenadoria de Assistência Técnica Integral- CATI, da Secretaria de Agricultura e Abastecimento e dá outras providências. São Paulo: Secretaria de Estado do Governo e Gestão Estratégica, 1997. Disponível em: <http://www.al.sp.gov.br/repositorio/legislacao/decreto/1997/decreto-41559-21.01.1997.html> Acesso em: set. 2016.

SÃO PAULO, Estado [de]. Secretaria de Agricultura e Abastecimento. Coordenadoria de Assistência Técnica Integral. Instituto de Economia Agrícola. Levantamento censitário de unidades de produção agrícola do Estado de São Paulo - LUPA 2007/2008. São Paulo: SAA/CATI/IEA, 2008. Disponível em: <http://www.cati. sp.gov.br/projetolupa>. Acesso em: jan. 2016.

SILVA, F. C. Agricultura familiar em duas microrregiões do noroeste do estado de São Paulo: uma análise comparativa entre as explorações agropecuárias e as políticas públicas dirigidas ao segmento. $137 f$. Dissertação (Mestrado em Agronomia) - Faculdade de Engenharia de Ilha Solteira, Universidade Estadual Paulista, Ilha Solteira, 2012.

SIMÕES, R. Localização industrial e relações intersetoriais: uma análise de "fuzzy cluster" para Minas Gerais. Tese (Doutorado em Economia) - Instituto de Economia, Universidade Estadual de Campinas, Campinas, São Paulo, 2003.

SNEATH, P. H.; SOKAL, R. R. Numerical taxonomy: the principles and practice of numerical classification. San Francisco: W. H. Freeman, 1973. 573p.

SOUSA, L. O.; CAMPOS, S. A. C.; GOMES, M. F. M. Technical performance of milk producers in the state of Goiás, Brazil, in the short and long terms. Revista Brasileira de Zootecnia, Viçosa, v. 41, n. 8, p. 19441950, ago. 2012.

SOUZA, P. C. T.; WILHELM, V. E. Uma introdução aos modelos DEA de eficiência técnica. TUIUTI: Ciência e Cultura, Curitiba, n. 42, p. 121-139, 2009.

STATSOFT, Inc. STATISTICA (data analysis software system), version 7, 2004. Disponível em: <www.statsoft. com>.

VIAN, C. E. F.; ANDRADE JÚNIOR, A. M. Evolução histórica da indústria de máquinas agrícolas no mundo: origens e tendências. In: CONGRESSO NACIONAL DE ECONOMIA E SOCIOLOGIA RURAL, 48., 25-28 jul. 2010, Campo Grande, MS. Anais... Campo Grande: SOBER, 2010.

\section{Sobre os autores:}

Jaqueline Bonfim de Carvalho: Mestre em Agronomia pela Universidade Estadual Paulista Júlio de Mesquita Filho (UNESP), Ilha Solteira, São Paulo, Brasil.E-mail: jaquecarvalho.agro@gmail.com

Alan Rodrigo Panosso: Professor Doutor, Departamento de Matemática, FEIS/UNESP. Área: Agronomia, com ênfase em ciência do solo, atuando principalmente nos seguintes temas: emissão de CO2 do solo (respiração do solo), geoestatística, fractais e análises multivariadas. 
E-mail: alanrp@mat.feis.unesp.br

Omar Jorge Sabbag: Professor Doutor, Departamento de Fitotecnia, Tecnologia de Alimentos, e Socioeconomia (DFTASE), FEIS/UNESP. Área: Economia e gestão do agronegócio. E-mail: sabbag@agr.feis.unesp.br

Maria Aparecida Anselmo Tarsitano: Professora Doutora, Departamento de Fitotecnia, Tecnologia de Alimentos, e Socioeconomia (DFTASE), FEIS/UNESP. Área: Ciências Agrárias, com ênfase em análises técnicas, socioeconômicas e ambientais na agropecuária. E-mail: maat@agr.feis.unesp.br 